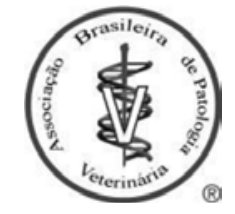

Diagnostic exercise From The Latin Comparative Pathology Group and

Diagnostic Exercise

From The Latin Comparative Pathology Group*

\title{
Ocular blastomycosis in a dog
}

Contributors:

Bianca S. de Cecco; ; Mariano Carossino ${ }^{2 * *}$; Pilar Camacho-Luna ${ }^{3}$; Christopher Alling;

Renee Carter $^{3}$; Jacqueline E. Elliott ${ }^{2}$; Fabio Del Piero ${ }^{2}$; Ingeborg M. Langohr ${ }^{2}$

${ }^{1}$ Department of Veterinary Pathology. Faculty of Veterinary Medicine, Federal University of Rio Grande do Sul, Porto Alegre, RS, Brazil.

${ }^{2}$ Louisiana Animal Diagnostic Disease Laboratory (LADDL) and Department of Pathobiological Sciences

School of Veterinary Medicine, Louisiana State University, Baton Rouge, LA, USA.

${ }^{3}$ Ophthalmology Service, Veterinary Teaching Hospital and Department of Veterinary Clinical Sciences,

School of Veterinary Medicine, Louisiana State University, Baton Rouge, LA, USA.

**Corresponding author: E-mail: mcarossino1@1su.edu

\section{Clinical History:}

A 2-year-old, female Poodle dog presented to the Emergency Service at the Veterinary Teaching Hospital (VTH), Louisiana State University School of Veterinary Medicine with a history of lethargy and anorexia. At clinical examination the dog was anemic and febrile. The dog was bilaterally blind. The right eye was buphthalmic and the intraocular pressure was elevated $(42 \mathrm{~mm} \mathrm{Hg})$. During an ophthalmological consult, bilateral severe panuveitis and optic neuritis with secondary glaucoma in the right eye was diagnosed and treatment was instituted. Despite treatment, the intraocular pressure kept increasing (up to $72 \mathrm{~mm} \mathrm{Hg}$ overnight), and the clinicians decided to enucleate the right eye due to poor prognosis. After one month, and no response to treatment, the left eye was also enucleated.

\section{Gross Findings:}

Right and left eyes: The vitreous chamber was filled with a yellow, gelatinous exudate while the anterior chamber was filled with a translucent, gelatinous fluid. The retina appeared detached and embedded within the exudate present in the vitreous chamber.

\section{Follow-up questions:}

- Microscopic description

- Morphologic diagnosis and cause

- $\quad$ Other possible causes

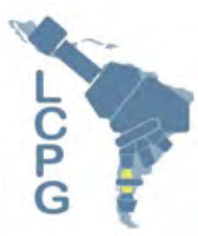

*The Diagnostic Exercises are an initiative of the Latin Comparative Pathology Group (LCPG), the Latin American subdivision of The Davis-Thompson Foundation and published in cooperation with the Brazilian Journal of Veterinary Pathology.

Editor-in-chief for this Diagnostic Exercise: Claudio Barros Associate Editor for this Diagnostic Exercise: Mariano Carossino

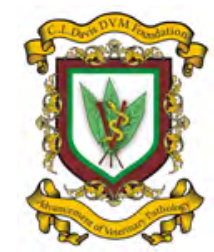



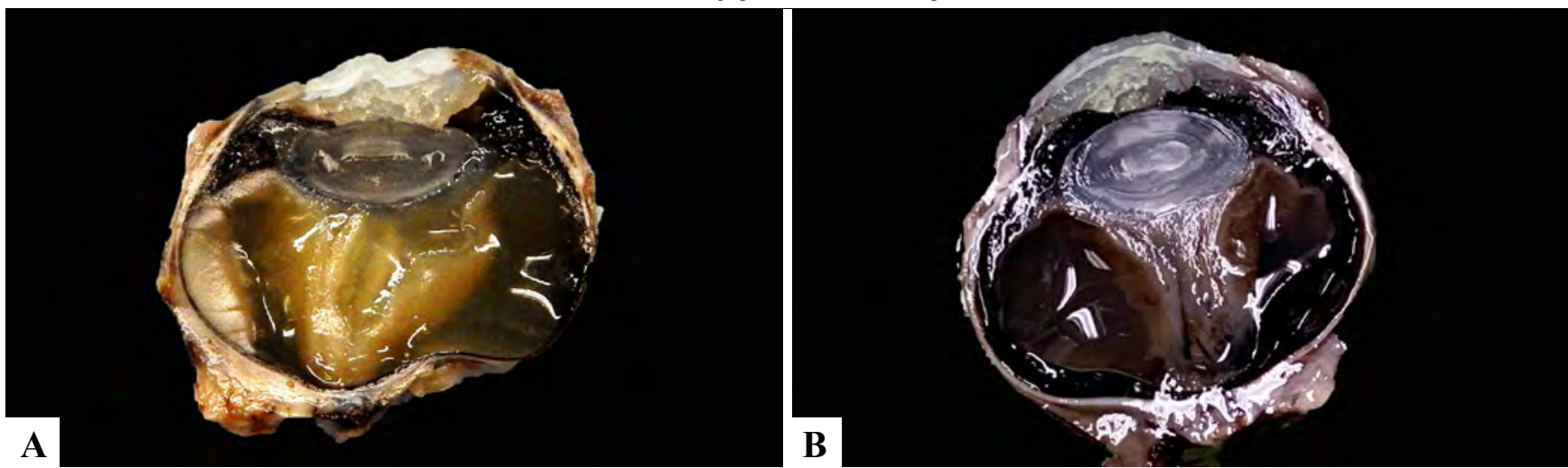

C

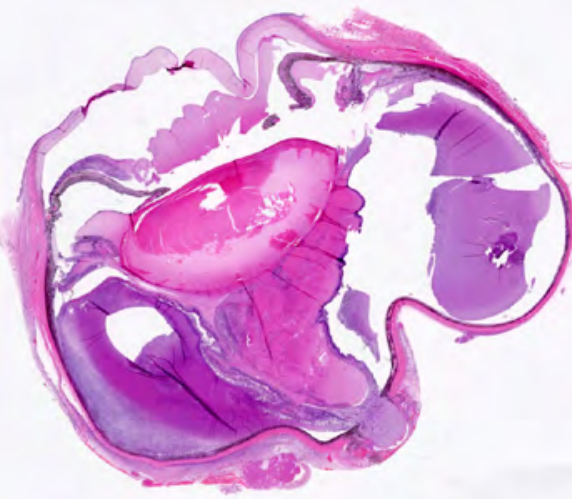

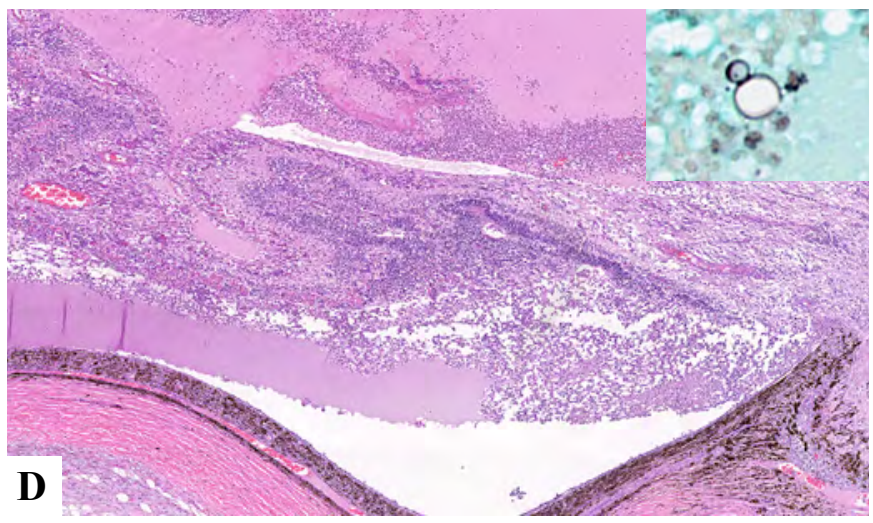

Figure 1. A. Right eye, bisected specimen. B. Left eye, bisected specimen. C. and D. Right eye, subgross and 40X, Hematoxylin and eosin. D. (Inset) Gomori methenamine silver stain. 40X.

Microscopic description: Microscopic changes in both eyes were similar but more severe in the right eye. The peripheral conjunctival epithelium is infiltrated by few neutrophils (exocytosis) with few dispersed neutrophils within the submucosa. The corneal stroma is mildly expanded by edema and infiltrated by few to low number of neutrophils, with multiple proliferating blood vessels at the periphery. The anterior chamber is filled with brightly eosinophilic fluid with numerous degenerate neutrophils and foamy histiocytes, and few melaninladen histiocytes. The inferior filtration angle contains low number of similar inflammatory cells. The inferior and superior iris leaflets are C-shaped, and their tip is adhered to the anterior capsule of the lens by organized fibrinous exudate mixed with degenerate neutrophils and histiocytes (iris bombé) (Fig. 1C). The anterior and posterior surfaces of the iris leaflets are coated by a thin pre and post-iridal fibrovascular membrane, and the stroma is infiltrated by few to low number of neutrophils and histiocytes. The ciliary body is markedly expanded by dense cellular aggregates composed of abundant neutrophils and histiocytes, fibrin and myxedema. The vitreous chamber is filled with abundant exudate similar to that noted in the anterior chamber, which contains rare intralesional yeast organisms (Fig. 1D - inset) measuring approximately 15 to $30 \mu \mathrm{m}$ in diameter. The yeast is characterized by a thick basophilic capsule, lightly basophilic to lightly eosinophilic granular content and broad-based budding, and highlighted by a Grocott's Methenamine Silver (GMS) stain (compatible with Blastomyces dermatitidis). The retinal pigment epithelium is segmentally plump with a "tombstoning" appearance (indicative of retinal detachment). The detached retina is embedded within the exudate contained in the vitreous chamber and is extensively disrupted by necrosis (Fig. 1D), characterized by loss of the normal layering pattern and ganglion cells, increased cellular eosinophilia, marked vacuolization of retinal layers and fibrin, accompanied by subretinal neutrophils and histiocytes. The choroid is diffusely and markedly expanded by dense cellular aggregates composed of neutrophils and histiocytes and, along its ventral aspect, the adjacent sclera is affected by liquefactive necrosis characterized by hyalinization and fragmentation of collagen fibers and abundant degenerate neutrophils. The optic nerve is infiltrated by low number of neutrophils and contains multifocal spheroids as well as dilated nerve fibers containing intralesional cell debris or Gitter cells. The lens is histologically unremarkable.

Morphologic diagnoses and cause: Panophthalmitis, pyogranulomatous, diffuse, severe, subacute, with retinal detachment, retinal necrosis, iris bombé, optic neuritis and rare intralesional yeasts consistent with Blastomyces dermatitidis (urine antigen test positive for $B$. dermatitidis). 


\section{Other possible causes:}

- Cryptococcus neoformans

- Histoplasma capsulatum

- Coccidioides immitis

- Leishmania sp.

\section{Discussion:}

Blastomyces dermatitidis is a saprophytic dimorphic fungus, that grows in sandy and acid soil, usually near water (Legendre, 2006; López-Martínez and Méndez-Tovar, 2012). It is considered a geographically restricted infectious agent distributed in North America, predominantly along the Ohio River Valley, Mississippi and Missouri (Schwartz, 2018). In the environment, this fungus reproduces sexually and produces infective spores (Legendre, 2006; Schwartz, 2018). At body temperature, these spores transform into yeasts and undergo an asexual reproduction phase (Legendre, 2006; Schwartz, 2018). This yeast infection is common in dogs and humans, and has also been sporadically described in several other domestic and wild animals, including cats, horses, wolves, ferrets, sea lions and other wild species (Legendre, 2006; Schwartz, 2018). Affected animals acquire the infective spores from the environment by inhalation of airborne conidia, and the primary infection is then established in the lungs; however, traumatic inoculation through the skin has also been described as a route of infection (Legendre, 2006; LópezMartínez and Méndez-Tovar, 2012; Schwartz, 2018). Dissemination to other organs subsequently occurs through both vascular and lymphatic routes (Legendre, 2006). The most common sites of dissemination include the skin and subcutis, eyes, brain, lymph nodes, and testes (Legendre, 2006). Clinical signs in dogs are usually nonspecific, and include fever, lymphadenopathy, cutaneous lesions, uveitis, and harsh lung sounds (Schwartz, 2018). Ocular involvement occurs in up to $50 \%$ of the cases (Grahn et al., 2019). Clinical and gross examinations frequently demonstrate endophthalmitis characterized by plasmoid aqueous and vitreous, retinal detachment, and swollen uvea (Grahn et al., 2019). Microscopically, the features observed in this case are similar to those described in the literature, including pyogranulomatous endophthalmitis, and extensive and severe subretinal exudates composed of neutrophils (Grahn et al., 2019). Yeasts can be identified within this exudate, which can be further highlighted using special stains, like periodic-acid Schiff reaction (PAS) and GMS (Legendre, 2006; López-Martínez and Méndez-Tovar, 2012). Yeasts measure from 5 to $20 \mu \mathrm{m}$ diameter and present a thick, refractile, double-contoured cell wall and characteristic broad-based budding that usually allows distinction from other dimorphic fungi and yeasts (Legendre, 2006; LópezMartínez and Méndez-Tovar, 2012; Grahn et al., 2019). While the identification of budding yeasts usually leads to its accurate diagnosis, occasionally yeasts are not identified in examined sections in which case, differential diagnoses for infectious causes of uveitis in dogs should include Cryptococcus neoformans, Histoplasma capsulatum, Leishmania spp., Coccidioides immitis, and other organisms like Ehrlichia canis, Toxoplasma gondii, and Prototheca spp. (Massa et al., 2002; Grahn et al., 2019).

Ocular cryptococcosis (caused by Cryptococcus neoformans and $C$. gattii; dimorphic, basidiomycetous fungi) is the main differential diagnosis in uveitis cases in dogs (Malik et al., 2006). Cryptococcus spp. has a worldwide distribution, and affects frequently young adult dogs, with nonspecific signs such as weight loss and lethargy (Malik et al., 2006). Ocular cryptococcosis can be differentiated from blastomycosis on $\mathrm{H} \& \mathrm{E}$ and special stains, since the inflammatory reaction is purely granulomatous, with a large number of encapsulated, poorly stained yeasts (giving the lesions a characteristic "soap-bubble" appearance) with narrow-based budding and positive staining with mucicarmine (Grahn et al., 2019).

Histoplasma capsulatum is a soil-borne, dimorphic fungus, endemic in temperate and subtropical regions, with high occurrence in central United States (Greene, 2006b). Young dogs are more susceptible, and clinical signs consist in inappetence, weight loss and fever (Greene, 2006b). The disease can be characterized as respiratory or disseminated (Greene, 2006b). It is considered to be a less frequent cause of endophthalmitis than other fungi (Grahn et al., 2019). This fungus causes primarily a choroiditis with exudative retinal detachment, microscopically characterized by proliferative and granulomatous reaction, with the organisms present in the cytoplasm of macrophages (Grahn et al., 2019). The visualization of the fungi is possible on $\mathrm{H} \& \mathrm{E}$, and can be differentiated from other fungi by their location (intracellular) and morphology: 2-4 $\mu \mathrm{m}$ spherical yeasts accentuated by a clear, unstained, peripheral halo (Grahn et al., 2019). Coccidioides immitis is another dimorphic fungus. This soil-borne fungus is restricted to certain geographic regions, known as Lower Sonoran life zone, characterized by sandy, alkaline soil with high environmental temperatures, including southwestern United States, Mexico, and Central and South America (Greene, 2006a). Most dogs positive for this fungus are asymptomatic; however, mild lower respiratory tract infection can be noticed (Greene, 2006a). Ocular lesions associated with this fungus include keratitis, uveitis and acute blindness. These lesions are usually similar to those seen in blastomycosis, are frequently equally distributed in both the anterior and posterior uvea, and have a greater tendency for granuloma formation (Grahn et al., 2019). Confirming the etiologic diagnosis based only on ocular changes is difficult, since the organisms are typically very sparse (Grahn et al., 2019).

Leishmaniasis is caused by diphasic protozoans of the genus Leishmania (Baneth, 2006). Clinical signs associated with this parasite are highly variable, including skin lesions, which are usually generalized, 
and visceral lesions accompanied by chronic disease with lymphadenomegaly and weight loss (Baneth, 2006). About $25 \%$ of the infected dogs will have ocular involvement, characterized mainly by mononuclear anterior uveitis (Pena et al., 2000). Additionally, adnexal lesions are common, and eyelid alopecia or other forms of blepharitis, and conjunctival inflammation are frequently associated with this organism (Pena et al., 2000). While each of these oculomycosis and leishmaniasis present a unique morphologic and characteristic histologic manifestations, all share similar clinical and histologic lesions (Grahn et al., 2019). A definitive diagnosis requires demonstration of the microorganism either directly via histology, cytology, and special stains, or indirectly by detection of antigen in ocular fluids or urine (Grahn et al., 2019).

The treatment implemented in this case was bilateral enucleation due to the severe panuveitis as well as oral itraconazole. Amphotericin B treatment is also a possible option; however, the risk of development of acute kidney injury is high. As already discussed, the primary organ affected by this dimorphic fungus is the lung. In this dog, a pulmonary granuloma was identified radiographically, which resolved during treatment. The dog was evaluated monthly and 5 months after testing positive for the first time for $B$. dermatitidis and treatment was implemented, the dog had a negative urine test result. Approximately 8 months after diagnosis, the treatment was discontinued with a favorable outcome.

\section{References}

1. Baneth G. 2006. Leishmaniases, p.685-696. In: Greene Infectious Diseases of the Dog and Cat. 3rd ed. Saunders Elsevier. St Louis, MO.

2. Greene R.T. 2006a. Coccidiomycosis and Paracoccidioidomycosis, 598-608. In: Greene Infectious Diseases of the Dog and Cat. 3rd ed. Saunders Elsevier. St Louis, MO.

3. Greene C.E. 2006b. Histoplasmosis, p.577-583. In: Greene Infectious Diseases of the Dog and Cat. 3rd ed. Saunders Elsevier. St Louis, MO.

4. Grahn B., Peiffer R., Wilcock B. 2019. Histologic Basis of Ocular Disease in Animals. 1st ed. John Wiley \& Sons. Hoboken, NJ. p.240-246.

5. Legendre A.M. 2006. Blastomycosis, p.569-576. In: Greene Infectious Diseases of the Dog and Cat. 3rd ed. Saunders Elsevier. St Louis, MO.

6. López-Martínez R., Méndez-Tovar L.J. 2012. Blastomycosis. Clin Dermatol 30:565-572.

7. Malik R., Krockenberger M., O'Brien C.R., Martin P., Wihney D., Medleau L. 2006. Cryptococcosis, p. 584598. In: Greene Infectious Diseases of the Dog and Cat. 3rd ed. Saunders Elsevier. St Louis, MO.

8. Massa K.L., Gilger B.C., Miller T.L., Davidson M.G. 2002. Causes of uveitis in dogs: 102 cases (19892000). Vet Ophthalmol. 5(2):93-98.
9. Pena M.T., Roura X., Davidson M.G. 2000. Ocular and periocular manifestations of leishmaniasis in dogs: 105 cases (1993-1998). Vet Ophthalmol 3:35-41.

10. Schwartz I.L. 2018. Blastomycosis in mammals, p. 159-176. In: Seyedmousavi S., Sybren de Hoog G., Guillot J., Verweij P.E. (Ed) Emerging and Epizootic Fungal Infections. Springer Nature. Switzerland. 